\title{
Pure-tone equal-loudness contours for standard tones of different frequencies
}

\author{
JOHN A. MOLINO* \\ Institute for Basic Standards, National Bureau of Standards, Washington, D.C. 20234
}

\begin{abstract}
Six Ss made judgments of equal loudness by adjusting the intensity of comparison tones of 10 different frequencies. The comparison tones were presented diotically alternately with standard tones. Each standard tone remained fixed at one frequency $(125,1,000$, or $8,000 \mathrm{~Hz})$ and one intensity $(10,20,40$, or $70 \mathrm{~dB}$ sensation level) while collecting the data for any single equal-loudness contour. In this manner, families of equal-loudness contours were generated for each of the three standard frequencies. The contours for the $1,000-\mathrm{Hz}$ standard were compared with those in the literature. The families of contours for the $125-$ and $8,000-\mathrm{Hz}$ standards, determined by the same algorithm, differed in the spacing of the contours from the $1,000-\mathrm{Hz}$ standard family as well as from each other. Implications for the reflexive, symmetric, and transitive properties of the equal-loudness relation are discussed.
\end{abstract}

Several determinations have been made of pure-tone equal-loudness contours elaborated in the classical manner. Kingsbury (1927) and Fletcher and Munson (1933) used earphones to present the sounds, whereas Churcher and King (1937) and Robinson and Dadson (1956) made measurements in a free acoustic field. The procedure for assessing the equal-loudness judgments of groups of Ss was similar in each case. One tone was selected as the standard or reference tone against which the loudness of other tones was compared. Either the standard tone (Kingsbury, Churcher, \& King) or the comparison tone (Fletcher \& Munson; Robinson \& Dadson) was held constant in intensity, while the intensity of the other was permitted to vary until equal-loudness levels could be ascertained. Except for Kingsbury (method of adjustment), the method of constant stimuli was used; however, the selection of a standard frequency was arbitrary in all cases. Kingsbury selected $700 \mathrm{~Hz}$ as his standard tone, whereas subsequent researchers have generally preferred $1,000 \mathrm{~Hz}$. In 1959 , the choice of $1,000 \mathrm{~Hz}$ as the reference tone for defining the subjective magnitudes of sound was endorsed by the International Organization for Standardization (ISO, 1959) in its Recommendation R131.

When elaborating meaningful equal-loudness contours across many frequencies, the use of a standard sound of a single arbitrary frequency makes certain assumptions. It is predicated upon the subjective relationship "sounds equally loud to" (L) possessing three important properties. If $S=\{$ all audible sounds $\}$ and $L \subseteq S \times S$, then one of these properties is reflexivity $\left(s_{1} L s_{1}\right)$. This first property was tested by Kingsbury by comparing his standard $700-\mathrm{Hz}$ tone with itself at different intensity levels. Although he found the variances to be rather

\footnotetext{
*The author gratefully acknowledges the conscientious assistance of Donna Free, Eudora Gray, and Linda Hawkins in executing this experiment.
}

small ( $2 \mathrm{~dB}$ on the average), this property was not tested by subsequent investigators. The second property of consequence is symmetry: $s_{1} L s_{2} \Rightarrow s_{2} L s_{1}$. Kingsbury examined the symmetry between equal loudness judgments at 700 and $1,000 \mathrm{~Hz}$ by interchanging the roles of the standard and comparison tones, and reported the discrepancies to be within the variability of his data. Robinson and Dadson performed a similar comparison for three pairs of tones $(33.3-50 \mathrm{~Hz}$, $50-200 \mathrm{~Hz}, 50-1,000 \mathrm{~Hz}$ ) and found errors within $1 \mathrm{~dB}$ on the average. The studies of Fletcher and Munson and of Churcher and King both assumed symmetry. The third property to be confirmed is transitivity, i.e., $s_{1} L s_{2}, s_{2} L s_{3} \Rightarrow s_{1} L s_{3}$. Although Fletcher and Munson merely assumed that their measurements were transitive, the other researchers actually put this property to empirical test. Kingsbury triangulated among 200,700 , and $3,200 \mathrm{~Hz}$, while Churcher and King compared 150,500 , and $1,000 \mathrm{~Hz}$ as well as 1,000 , 2,500 , and $4,500 \mathrm{~Hz}$, and Robinson and Dadson experimented with several combinations of frequencies. These authors argued that if two tones were judged as equally loud to the standard, then they should be judged as equally loud relative to each other. Through such intercomparisons, they found transitivity to be a property of the equal-loudness relation within the probable error of their measurements.

In addition to the classical studies mentioned above, there have been several recent investigations of equal-loudness relations, but these studies have had a somewhat different emphasis. Although based upon an empirically tested symmetric and transitive set of data, the carefully executed study of Ross (1967) was directed at measuring loudness functions for different individuals. Gerber and Milner (1971) confirmed the symmetry and transitivity of a single loudness level, but they used octave bands of noise rather than pure tones as stimuli. While the experiment of Schneider et al (1972) provides a unique look at the shape of equal-loudness contours, it is based upon magnitude 


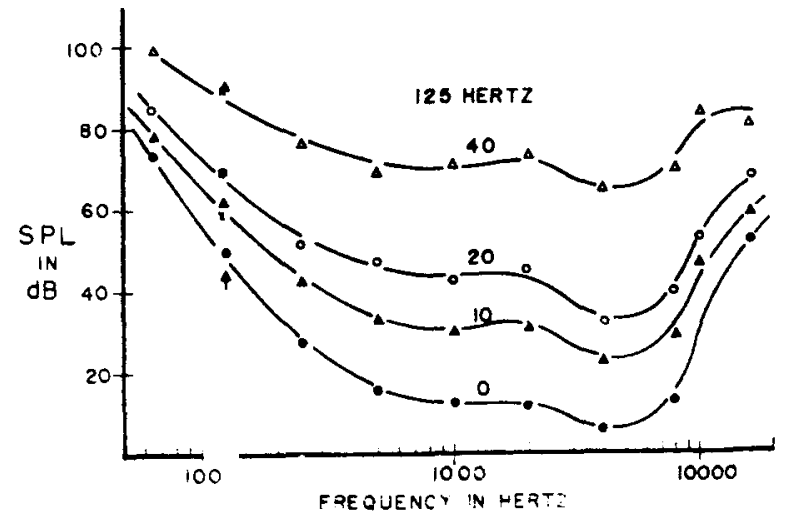

Fig. 1. Pure-tone equal-loudness contours for a $125-\mathrm{Hz}$ standard frequency.

estimation instead of equal-loudness judgments. It is the purpose of the present study. however, to remain within the framework of the classical procedure for determining equal-loudness contours, except that the arbitrary parameter of standard tone frequency is varied in order to produce not one, but three families of equal-loudness contours.

\section{METHOD}

\section{Subjects}

The Ss were six high school students between the ages of 16 and 19 years. Each $S$ was otologically normal, as tested with continuous pure tones on a Békésy-type recording attenuator. Thresholds were referred to International Organization for Standardization (ISO. 1964) R/389.

\section{Apparatus}

The standard and comparison tones were generated by separate oscillators and alternately gated on for $1 \mathrm{sec}$ and off for 1 sec by separate electronic switches. The rise and fall times were $100 \mathrm{msec}$. There was a $150-\mathrm{msec}$ silent period between alternating tone bursts. during which time separate mercury-wetted relays alternately switched in first the standard channel and then the comparison channel to a pair of Beyers DT 48-S earphones. The relays insured that the background noise from one channel would not contaminate the signal in the other channel, thus maintaining an overall $\mathrm{S} / \mathrm{N}$ ratic of $60 \mathrm{~dB}$ at all times. Since the relays were switched only during the silent period, they did not contribute any appreciable transient sound. Each channel was isolated from the earphones by appropriate control attenuators located between the relays and the earphones. In addition, the comparison tone channel had a recording attenuator inserted at this point. The recording attenuator was under the S's control so that he could increase or decrease the intensity of the comparison tone at the rate of $4 \mathrm{~dB} / \mathrm{sec}$ or let it remain constant. The rms voltage across each earphone was monitored and maintained equal to within $0.5 \mathrm{~dB}$. The earphones were calibrated in an NBS-9A coupler, with the PTB adapter especially designed for that coupler. Although listening was diotic, the sound pressure levels (SPL) reported are for a single phone. Throughout the experiment, the $S$ sat in a sound-attenuating chamber meeting American National Standards Institute (ANSI. 1960) S3.1 requirements.

\section{Procedure}

The Ss were read instructions explaining that they were to adjust the comparison tone until it "sounds equally loud to" the standard tone. Before each session, a standard tone of one frequency $(125,1.000$, or $8.000 \mathrm{~Hz})$ was selected and two brief determinations of the $S$ 's absolute threshold at that frequency were made by the method of adjustment. For these measurements, the standard tone was delivered through the comparison tone channel and the signal in the standard tone channel was attenuated well below threshold. After the absolute threshold had been secured, the standard tone was restored to its normal channel and presented at a preselected sensation level (10, 20, 40. or $70 \mathrm{~dB}$ ) above the absolute threshold just determined. At the same time, one of 10 frequencies (including the standard tone frequency) was selected and delivered over the comparison tone channel. The $\mathrm{S}$ listened to the alternating sequence of standard and comparison tones and adjusted the comparison tone until he had achieved a loudness match. Next. after an arbitrary amount of attenuation had been inserted into the comparison channel. the $\mathrm{S}$ made a second loudness match. Then the frequency of the comparison tone was changed and two equal-loudness judgments were secured in the same manner at the new frequency. The procedure was repeated until 20 judgments had been made (two at each frequency) in the course of a 20-min session. The order of presenting the comparison frequencies was such that tones requiring higher intensities for a loudness match were presented toward the end of the session. so as to minimize adaptation effects. Each $\mathrm{S}$ completed $10 \mathrm{such}$ sessions, each session providing the data for one equal-loudness contour.

\section{RESULTS}

The results of the experiment are shown in Figs. 1-3 for the three standard frequencies. The $0-\mathrm{dB}$ contour represents the average absolute threshold for the group of six Ss and is the same for each figure. The standard errors for the absolute threshold measurements ranged from 1.8 to $5.9 \mathrm{~dB}$, with a mean value of $2.9 \mathrm{~dB}$. The data points on the $10-70-\mathrm{dB}$ contours represent the means of 12 equal-loudness matches for the six Ss. The standard errors for the loudness matches ranged from 1.1 to $8.2 \mathrm{~dB}$, with a mean value of $3.8 \mathrm{~dB}$. The vertical arrow indicates the standard frequency being employed for each figure, while the crosses indicate the average intensity levels of the standard tone employed for the various contours. The $70-\mathrm{dB}$ equal-loudness contour could not be measured with a 125 - and $8,000-\mathrm{Hz}$ standard tone due to limitations in generating undistorted acoustic stimuli at extremely high intensity levels.

The curves through the data points were fitted by eye. These curves were compared with those obtained by two other curve-fitting methods. In accordance with the procedure outlined by Fletcher and Munson (1933, p. 90), smooth curves were drawn by eye through the appropriate loudness growth functions, and the corresponding SPL values were read from the growth function plots in order to generate another family of curves through the same data points. Secondly, the data points up to $8,000 \mathrm{~Hz}$ were fitted by least squares to a two-dimensional polynomial of the form 


$$
S P L=\Sigma a_{i j} L^{i} f^{j},
$$

with $\mathrm{i}$ ranging from 0 to 2 , and $\mathrm{j}$ from 0 to $5 .{ }^{1}$ When compared with the original curves depicted in Figs. 1.3, the results of both of these alternative methods for fitting equal-loudness contours revealed essentially the same features. In one region on the $1,000 \cdot \mathrm{Hz}$ standard contours, between 2,000 and $3,000 \mathrm{~Hz}$, the maximum divergence was $6 \mathrm{~dB}$. Otherwise, the various families of contours for a given standard frequency fitted by the three different methods all fell within a maximum discrepancy of $4 \mathrm{~dB}$ and were in many places indistinguishably different.

\section{DISCUSSION}

The family of contours for the $1,000-\mathrm{Hz}$ standard may be compared with others found in the literature. As concerns the SPL for the various contours, as opposed to their shape, the present data compare best with those of Kingsbury (1927). The absolute threshold curve of the present study ( 0 -dB contour) agrees with that of Kingsbury as well as with that recommended in ISO/R389 to within a maximum discrepancy of $4 \mathrm{~dB}$ over the range from 150 to $4,000 \mathrm{~Hz}$, the upper limit of Kingsbury's measurements. At 125 and $8,000 \mathrm{~Hz}$, the absolute threshold curve of the present study is within $2 \mathrm{~dB}$ of the ISO value, whereas the Kingsbury data begin to diverge at frequencies below $150 \mathrm{~Hz}$-in terms of both absolute threshold and equal-loudness contours. Comparison with the equal-loudness contours of Fletcher and Munson (1933), the other classically derived contours for earphone listening, is not as simple. It necessitates a level shift of $+10 \mathrm{~dB}$ to bring the absolute threshold curve of Fletcher and Munson into closer correspondence with those of Kingsbury, the ISO, and the present study. This shift having been made, the Fletcher and Munson contours are rather similar to the present set in both shape and level from 200 to $4,000 \mathrm{~Hz}$, with the exception of the exaggerated bulge in the present 10 - and $20-\mathrm{dB}$ contours at $2,000 \mathrm{~Hz}$ (the same region where maximum disagreement among curve fitting procedures was encountered). At the higher and lower frequencies, the divergences are extensive.

When the earphone-derived contours of the present experiment are compared with the free-field equal-loudness contours of Churcher and King (1937) and Robinson and Dadson (1956), even with corrections made for differences in absolute threshold, the correspondence is in general not very close. On the other hand, when the present 70-dB contour is compared with the appropriate "characteristic contours" of Ross (1967) and Schneider et al (1972), there is an excellent correspondence $( \pm 3 \mathrm{~dB})$ between mean values up to $2,500 \mathrm{~Hz}$ (the upper frequency limit of Schneider et al), except for slight deviations at $100 \mathrm{~Hz}$ and between 800 and $1,250 \mathrm{~Hz}$. This $\pm 3 \cdot \mathrm{dB}$ agreement extends down to $63 \mathrm{~Hz}$, where the contours of the present experiment diverge most from the more shallow slopes found in the

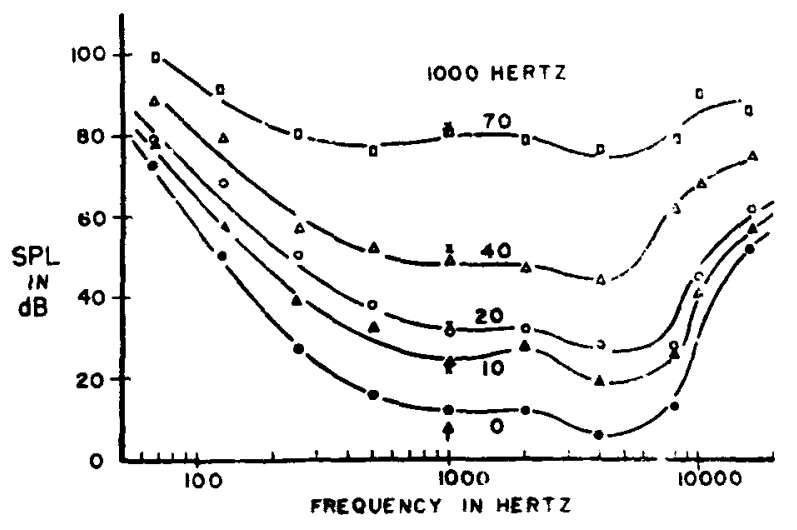

Fig. 2. Pure-tone equaltoudness contours for a $1,000-\mathrm{Hz}$ standard frequency.

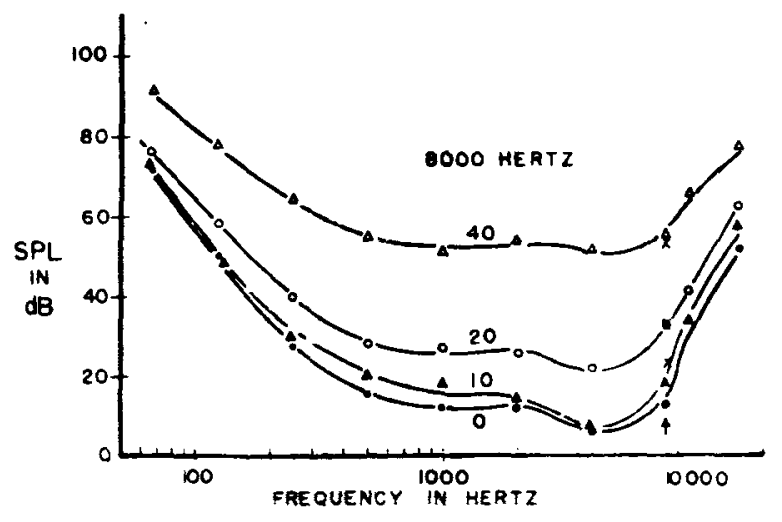

Fig. 3. Pure-tone equal-loudness contours for an $8,000-\mathrm{Hz}$ standard frequency.

four classical investigations cited earlier. In this low-frequency region, the data of the present study are in better accord with the recent determinations of individual equal-loudness contours of Ross and Schneider et al, possibly due to reduced harmonic distortion in the signals. Ross maintained less than $1 \%$ distortion in his tones, and attributed the steeper slope of his contours at lower frequencies to possible impurities in the stimuli of earlier investigators.

In addition to comparison with curves found in the literature, the present experiment permits the comparison of families of equal-loudness contours elaborated by the same Ss with three different standard frequencies, as shown in Figs. 1-3. When these families of curves are intercompared, a distinct difference is evident in the spacing of contours within each family. Those for the $125-\mathrm{Hz}$ standard are more spread apart; those for the $1,000-\mathrm{Hz}$ standard are intermediately spaced; and those for the $8,000-\mathrm{Hz}$ standard are more compressed at the lower intensity levels. These changes in spread, however, do not necessarily imply any violations of the reflexive, symmetric, and transitive properties of the equal-loudness relation as classically defined. In order for significant violations to 


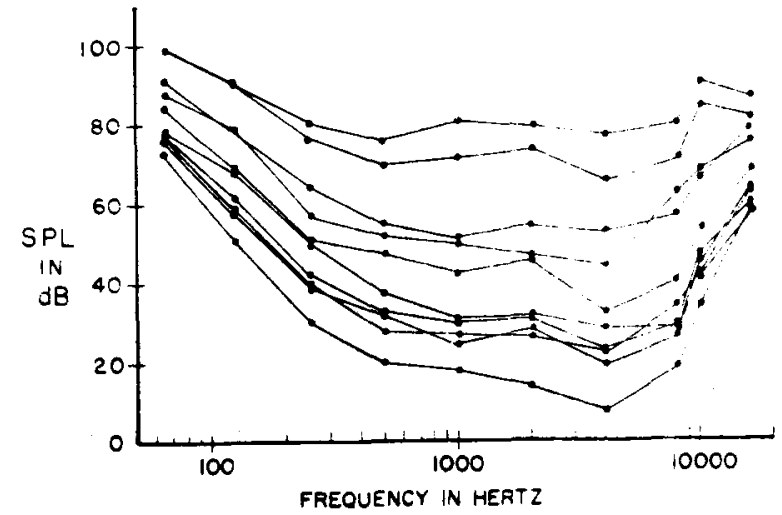

Fig. 4. Pure-tone equal-loudness data for three standard frequencies. The threshold curve has been omitted.

occur, the contours would have to be different in shape, as distinct from spread, for each of the standard frequencies. Although the results of the present experiment permitted some direct intercomparisons for symmetry and transitivity, producing discrepancies as great as $10 \mathrm{~dB}$. no definite trends emerged in the shapes of the equal-loudness contours. In Fig. 4 , all the equal-loudness measurements. irrespective of standard frequency, are plotted by simply connecting the data points by straight lines without any curve fitting procedure. From this figure, it is extremely difficult to distinguish consistent differences in the shapes of the contours measured with different standard frequencies. To the extent that no consistent differences could be observed within the variability of the present data, symmetry and transitivity appear to be properties of the equal-loudness relation.

As for the reflexivity assumption, the inclusion in the experimental design of comparison tones of the same frequency as the standard frequency afforded 10 opportunities to test this property. In all cases, the discrepancy was less than $3 \mathrm{~dB}$, except for the $8,000-\mathrm{Hz}$ standard $10-\mathrm{dB}$ point, where it was $7 \mathrm{~dB}$. For all but the latter instance, the differences were well within the standard error of the measurements.

\section{REFERENCES}

ANSI. American standard criteria for background noise in audiometer rooms. American National Standards Institute. S3.1, 1960.

Churcher, B. G., \& King, A. J. The performance of noise meters in terms of the primary standard. Journal of the Institution of Electrical Engineers, 1937, 81, 57-90.

Fletcher, H., \& Munson, W. A. Loudness, its definition, measurement, and calculation. Journal of the Acoustical Society of America. 1933, 5, 82-108.

Gerber, S. E., \& Milner, P. The transitivity of loudness level Journal of the Audio Engineering Society, 1971, 19, 656-659.

ISO. Expression of the physical and subjective magnitudes of sound and noise. International Organization for Standardization, R/131, 1959.

ISO. Standard reference zero for the calibration of pure-tone audiometers. International Organization for Standardization, $\mathrm{R} / 389.1964$.

Kingsbury, B. A. A direct comparison of the loudness of pure tones. Physics Review, 1927, 29. 588-600.

Robinson, D. W., \& Dadson, M. A. A re-determination of the equal-loudness relations for pure tones. British Journal of Applied Physics, 1956, 7, 166-181.

Ross, S. Matching functions and equal-sensation contours for loudness. Journal of the Acoustical Society of America, 1967. $42,778-793$.

Schneider, B.. Wright, A. A.. Edelheit, W., Hock, P.. \& Humphrey, C. Equal loudness contours derived from sensory magnitude judgments. Journal of the Acoustical Society of America, 1972, 51, 1951-1959.

\section{NOTE}

1. This method was suggested by Martin Greenspan. Sound Section, National Bureau of Standards. He developed the equations and assisted in the computations.

(Received for publication December 1, 1972; revision received February $21,1973$. ) 